\title{
Deposition-free Cryo-FIB Lift-out Transfer for Cryo-Electron Tomography Specimen Preparation
}

Jürgen Plitzko ${ }^{1}$, Philipp Erdmann ${ }^{2}$ and Sven Klumpe ${ }^{3}$

${ }^{1}$ Max Planck Institute of Biochemistry, Martinsried, Germany, United States, ${ }^{2}$ Fondazione Human

Technopole, Milan, Italy, United States, ${ }^{3}$ Max-Planck-Insitute for Biochemistry, United States

Recently, it has been demonstrated that cryo-focused ion beam milling (cryo-FIB) can be used to obtain high-quality samples from complex cellular environments for cryo-electron tomography (cryo-ET) from both plunge-frozen and high pressure frozen (HPF) cells, organisms and tissues [1-3]. These advances revealed the inner organization of cells and tissues at the molecular scale and at unprecedented resolution, coining the term "molecular sociology" [4].

Although technically demanding and involving time-consuming preparation steps, the extraction of lamellas from large high-pressure frozen samples (lift-out) can be successfully conducted using two different approaches $[1,5,6]$ :

The first approach uses a cryo-gripper to transfer the volume of interest from a bulk sample to a receiver grid with slots previously made by FIB milling. However, the preparation of these receptacle grids is a time-consuming process. Moreover, the receiver grid with the delicate lamella needs to be mounted in cartridges for the subsequent transfer to the TEM, with the risk of damaging or even losing the lamella [1].

The second approach to extract lamellae from bulk HPF material employs a sharp tungsten needle. Here, the transfer to a receiver grid is achieved by depositing platinum via a gas injection system (GIS) that attaches the needle to the volume of interest. Since under cryo-conditions the platinum is deposited over the entire sample, the resulting platinum layer will accumulate with each lift-out, thus limiting the total number of transfers that can be performed [7].

In this work, we demonstrate the application of deposition-free cryo-FIB lift-out using both the gripper and the needle system. While the gripper does not require local material deposition for the transfer to the receiving grid, the needle is attached by re-depositing needle material onto the volume of interest, thereby avoiding the build-up of a platinum layer. For both gripper and needle, extracted volumes are then welded onto a half-moon grid that has been pre-assembled in a cryo-EM cartridge. This is again achieved using a re-deposition procedure similar to that described previously [7] before fine-milling and transfer to the TEM. Circumventing the cartridge assembly after lift-out greatly enhances the success rate. While contamination during transfer between cryo-FIB and TEM still limits the accessible positions for tomography, the resulting lamella quality is equivalent to standard on-the-grid milling approaches. 

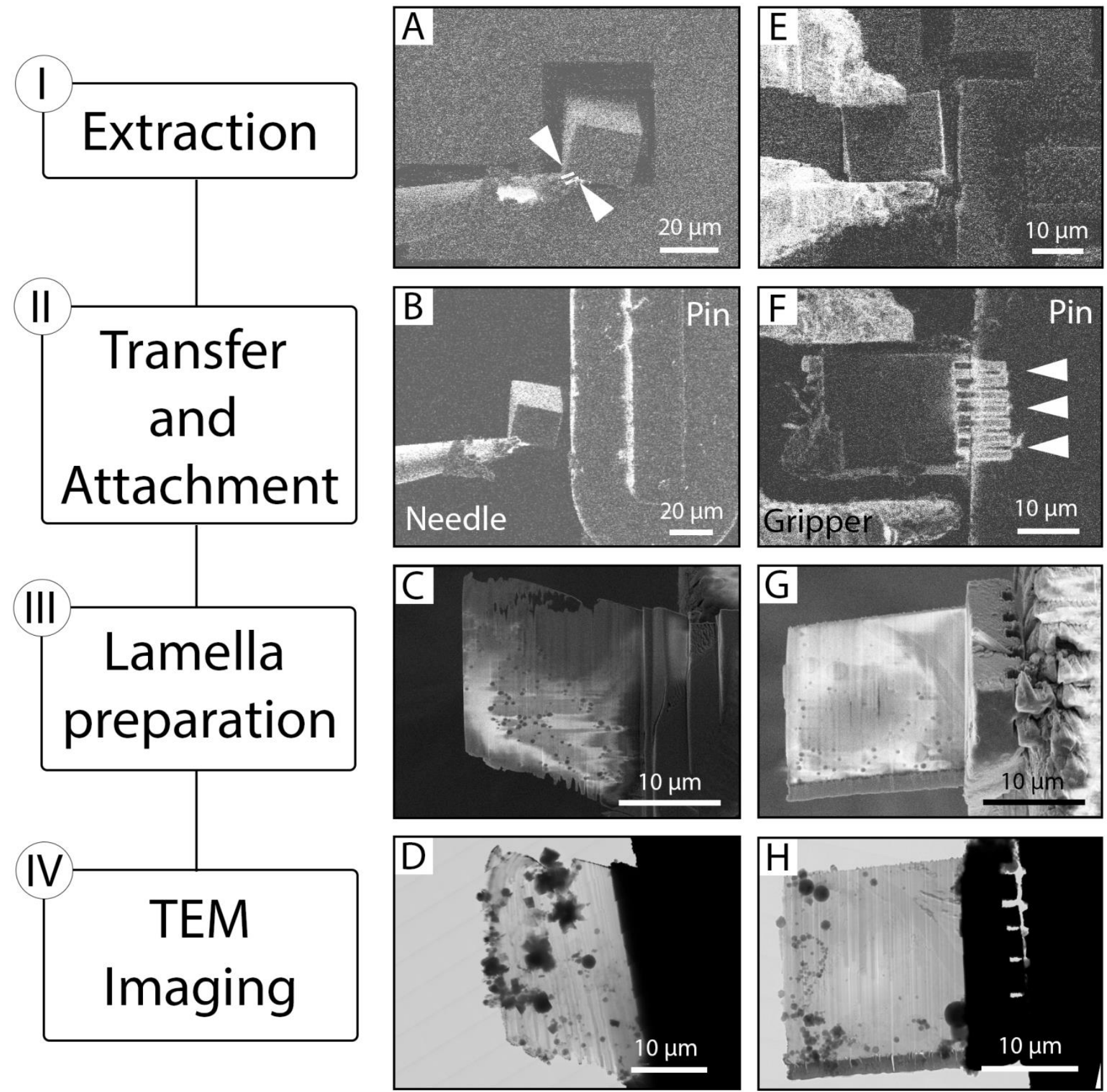

Figure 1. Figure 1: Cryo-FIB lift-out workflow. I: A volume is extracted from the bulk by either A: attachment to a tungsten needle by re-deposition or E: a cryo-gripper. Arrowheads in A indicate milling region for re-deposition. II: B: Transfer to a half-moon grid and F: attachment by re-deposition of halfmoon grid material through milling regions indicated by arrowheads. III: Scanning electron microscopy (SEM) overview of a polished lamella produced by C: the needle and G: the gripper lift-out approach. IV: TEM overviews of the lamellae produced by $\mathrm{D}$ : the needle and $\mathrm{H}$ : the gripper approach.

References

[1] M Schaffer et al., Nature Methods 2019. 16(8): 757-762

[2] A Rigort et al., PNAS 2012. 109(12): 4449-4454 
[3] M Marko et al., Journal of Microscopy 2006. 222(Pt 1): 42-47

[4] M Beck \& W Baumeister, Trends in Cell Biology 2016. 26(11): 825-837

[5] J Mahamid et al., Journal of Structural Biology 2015. 192(2): 262-269

[6] C D Parmenter \& Z A Nizamudeen, Journal of Microscopy 2020. 281(2): 157-174

[7] J Kuba et al., Journal of Microscopy 2021. 281(2): 112-124 